\title{
Expression Profiling of Circular RNAs and Micrornas in Heart Tissue of Mice with Alcoholic Cardiomyopathy
}

\author{
Yuqiao Yang ${ }^{a}$ Hongmei Chen ${ }^{b}$ Nina Ding ${ }^{a}$ Shuo Wang ${ }^{a}$ Zhantao Duan ${ }^{a}$ \\ Yochai Birnbaum ${ }^{c}$ Yumei Ye $^{\mathrm{d}}$ Jinqiao Qian ${ }^{\mathrm{a}}$ \\ aDepartment of Anesthesiology, First Affiliated Hospital of Kunming Medical University, Kunming, \\ 'Department of Anesthesiology, Kunming Angel Women's and Children's Hospital, Kunming, China, \\ 'Department of Medicine, Section of Cardiology, Baylor College of Medicine, One Baylor Plaza, \\ Houston, dDepartment of Biochemistry and Molecular Biology, University of Texas Medical Branch, \\ Galveston, USA
}

\section{Key Words}

Alcoholic cardiomyopathy (ACM) • Non-coding RNA • Competing endogenous RNA • Circular RNAs • MicroRNAs

\begin{abstract}
Background/Aims: Chronic heavy alcohol consumption may result in alcoholic cardiomyopathy. This study was designed to screen differentially expressed microRNAs and circular RNAs in heart tissue of mice with alcoholic cardiomyopathy to reveal the underlying molecular mechanism. Methods: Having established a murine alcoholic cardiomyopathy model, we screened differentially expressed microRNAs and circular RNAs in three heart samples from the alcohol-treated and control groups by high-throughput microarray analysis. We analyzed the function and biological signaling pathways of differentially expressed non-coding RNAs closely related to alcoholic cardiomyopathy using bioinformatics software to identify some mRNAs and their biological signaling pathways closely related to alcoholic cardiomyopathy. Results: Nineteen microRNAs and 265 circular RNAs were differentially expressed in the alcohol-treated group compared with the control group. After analyzing gene function and signaling pathways by bioinformatics software, we found that the differentially expressed mRNAs were associated with carbohydrate metabolism. Conclusions: Chronic alcohol consumption can change the non-coding RNA profile of heart tissue, which is closely related to the pathological mechanisms of alcoholic cardiomyopathy.

\section{Introduction}

Alcoholic cardiomyopathy (ACM) is a specific myocardial disease found in patients with a long history of heavy alcohol consumption. Chronic alcohol intake contributes to the onset Y. Yang, H. Cheng and N. Ding contributed equally to this work.


of a range of adverse cellular, histological, and structural changes in cardiomyocytes, which are characterized by normal or reduced left ventricular (LV) wall thickness, increased LV mass, LV dilatation, and reduced LV ejection fraction. No specific immunohistochemical biomarkers or other criteria for the diagnosis of ACM are available; therefore, its diagnosis is based on presumption, deteriorating cardiac function, and chronic alcohol abuse. ACM is always at an advanced stage when it is diagnosed. This devastating disease is one of the most prevalent causes of non-ischemic sudden cardiac death [1]. Although some studies have suggested the pathogenesis of ACM, including mitochondrial damage [2], cardiotoxicity of alcohol, oxidative stress [3-5], and the accumulation of fatty acid ethyl esters [6], the real pathological factors remain unknown. Genetic regulation plays an important role in the occurrence of ACM. To date, investigations into the molecular mechanisms of ACM development have not been reported.

Recently, non-coding RNAs have emerged as powerful regulators of biological processes, providing further opportunities for a better understanding of the biological mechanisms underlying the development of cardiovascular diseases [7]. MicroRNAs (miRNAs) are a class of well-studied non-coding RNAs that are 21-25 nucleotides long and capable of negatively regulating target mRNA at the post-transcriptional level. They repress mRNA translation or degrade target gene mRNA through sequence-specific interactions with the 3 '-untranslated region of target mRNA [8]. Functionally, miRNAs play a role in embryonic development, cell differentiation, apoptosis, growth, and the occurrence and development of many diseases and cancers. Additionally, miRNAs have both powerful and fine regulatory function in cardiovascular diseases and are associated with heart development, heart and arterial remodeling, vessel degeneration, and a series of cardiovascular diseases such as cardiomyopathy, coronary artery disease, and congestive heart failure [7]. Recent evidence has demonstrated that miRNA activity can be affected by the presence of miRNA "sponge" transcripts, also termed competing endogenous RNAs (ceRNAs), which include miRNAs and other elements of the transcriptome, such as pseudogenes, protein coding genes, circular RNAs (circRNAs), and long non-coding RNAs [9]. All types of RNA transcripts engage in cross-talk through the "language" mediated by miRNA binding sites, termed miRNA response elements (MREs) [10], which inhibit normal miRNA targeting activity on mRNA.

As newcomers to the ceRNA network, circRNAs are characterized by their abundant, conserved, stable, and tissue/developmental stage-specific expression $[11,12]$. In the past few years, circRNAs have generally been considered to be of low abundance and to likely represent splicing errors. No known function has been ascribed to endogenous circRNA transcripts. However, this picture has changed dramatically over the last 3 years. It has been demonstrated that circRNAs regulate gene expression in mammals [11,13-15]. Additionally, these species have been typically identified as a substantial fraction of the transcriptional output of cells [15]. They have an unusual structure, with 3 ' sequences located in a 5' position, lack poly(A) tails, preferentially localize to the cytoplasm, and enjoy extraordinary stability compared to linear RNAs owing to their ability to resist digestion by the exonuclease RNase $\mathrm{R}[13,14]$.

CircRNAs include exons from non-coding or coding transcripts spliced in an unconventional order $[11,13,15,16]$. The exons retained in circRNAs add an additional layer of complexity to the circRNA transcriptome. For example, some recently identified circRNAs can actually bind to host miRNAs, which are a class of important post-transcriptional regulators of protein-coding gene expression; such depletion of available miRNAs appears to have important biological roles, including homeostasis and the development of cardiovascular diseases and cancers $[12,17]$. For instance, Sry RNA is a male sex-determining gene and its circular isoforms are highly expressed in adult testis [16]. Sry has 16 binding sites for miRNA (miR)-138, and its expression represses miR-138 activity [12]. Another well-known circRNA is ciRS-7 (also termed CDR1as), which is derived from an antisense transcript of the cerebellum degeneration-related antigen 1 protein-coding gene, which has the potential to inhibit miR-7 activity and increase the levels of miR-7 targets [12]; cANRIL 


\section{Cellular Physiology Cell Physiol Biochem 2018;46:2284-2296

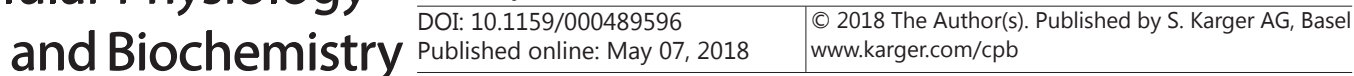

Yang et al.: Circular RNAs and MicroRNAs Profile in ACM Mouse Hearts

is an exceptionally long $100-\mathrm{kb}$ non-coding RNA transcript that also exists as a circular structure and is associated with an increased risk for atherosclerosis [18].

In this study, a model of ACM was established in mice after 24 weeks of alcohol consumption. We profiled the expression of circRNAs and miRNAs in the hearts of the experimental and control groups using microarray analysis. We used bioinformatics software to analyze the relationship between circRNAs and miRNAs and to predict putative miRNAtarget sites of circRNAs.

\section{Materials and Methods}

\section{Experimental animals and ethics approval}

Ten-week-old male Kunming mice were provided by the Experimental Animal Center of Kunming Medical University. The care of the experimental animals and all experimental handing procedures were in accordance with Chinese animal protection laws, and the Ethics Committee of Kunming Medical University specifically approved this study.

\section{ACM model}

Sixty Kunming mice were kept in a temperature-controlled room under a $12 \mathrm{~h} / 12 \mathrm{~h}$-light/dark cycle, allowed access to tap water, and fed with nutritionally complete ordinary diet ad libitum for a 1-week acclimation period. Upon completion of the acclimation period, 30 of the Kunming mice were maintained on regular tap water and ordinary diet, and the remaining 30 began a 24-week period of 4\% (vol/vol) alcohol consumption ad libitum. All animals were housed individually in standard plastic cages. Their body weights were recorded biweekly until the final week before sacrifice. After 24 weeks, the animals were sacrificed by cervical dislocation. The left side of the chest was opened and the heart was removed with surgical scissors, weighed immediately, and immersed in $10 \%$ neutral buffered formalin for hematoxylin and eosin (H\&E) staining. Histological changes of heart tissue were observed under a light microscope.

\section{Preparation and examination of ultra-thin sections}

The LV myocardium was cut into small tissue blocks ( $\left.\leq 2 \mathrm{~mm}^{3}\right)$, which were fixed with $3.5 \%$ glutaraldehyde at $4^{\circ} \mathrm{C}$ overnight. The blocks were rinsed with $0.2 \mathrm{M}$ phosphate buffer (pH 7.4), fixed in $1 \%$ osmium tetroxide for 1-2 h, and rinsed with distilled water for $30 \mathrm{~min}$. The blocks were dehydrated with graded ethanol and acetone and then embedded in Araldite Epon 618. Semi-thin sections (1-2 $\mu \mathrm{m}$ thick) were made from the blocks with a glass knife and stained with toluidine blue and H\&E and examined under a light microscope for selection. Ultra-thin sections (70-80 nm thick, silver-gray interference color) were prepared and stained with $1 \%$ uranyl acetate and $0.4 \%$ lead citrate. These sections were examined under a transmission electron microscope.

\section{Samples and RNA extraction}

After confirmation of ACM in the alcohol-treated mice, 3 mice were chosen randomly from each group by cervical dislocation. The heart was removed immediately from each mouse, and the LV wall was incised, frozen quickly in liquid nitrogen, and transported on dry ice to KangChen Bio-tech (Shanghai, PR China) for microarray analysis.

Total RNA was extracted from mouse ventricular muscle samples with the TRIzol reagent (Invitrogen, Carlsbad, CA, USA) and purified using an RNeasy Mini Kit (QIAGEN, Hilden, Germany) according to the manufacturers' instructions. Total RNA from each sample was qualified and quantified with a NanoDrop spectrophotometer (ND-1000; NanoDrop Technologies, Wilmington, DE, USA). RNA integrity was assessed by using standard denaturing agarose gel electrophoresis.

\section{CircRNA labeling and array hybridization}

RNA array hybridization and labeling were performed according to the manufacturer's protocol (Arraystar, Inc., Rockville, MD, USA). Briefly, circRNAs were treated with RNase R (Epicentre, Inc., Madison, WI, USA) to remove linear RNAs. Then, the samples were amplified and transcribed into fluorescent cRNAs by using a random priming method, and the labeled cRNAs were purified with an RNeasy Mini Kit (QIAGEN).

\section{KARGER}




\section{Cellular Physiology Cell Physiol Biochem 2018;46:2284-2296 \\ \begin{tabular}{c|c|c|} 
DOI: 10.1159/000489596 & $\begin{array}{l}\text { O } 2018 \text { The Author(s). Published by S. Karger AG, Basel } \\
\text { www.karger.com/cpb }\end{array}$
\end{tabular}}

Yang et al.: Circular RNAs and MicroRNAs Profile in ACM Mouse Hearts

The concentration and specific activity of the labeled cRNAs (pmol Cy3/ $\mu \mathrm{g}$ cRNA) was measured using a NanoDrop ND-1000. One microgram of each labeled cRNA was fragmented by adding $5 \mu \mathrm{L}$ of $10 \times$ blocking agent and $1 \mu \mathrm{L}$ of $25 \times$ fragmentation buffer, and the mixture was heated at $60^{\circ} \mathrm{C}$ for $30 \mathrm{~min}$. Finally, $25 \mu \mathrm{L}$ of $2 \times$ hybridization buffer was added to dilute the labeled cRNA. Hybridization solution $(50 \mu \mathrm{L})$ was added to the gasket slide and the circRNA expression microarray slide was assembled. The slide was incubated for 17 $\mathrm{h}$ at $65^{\circ} \mathrm{C}$ in an Agilent Hybridization Oven. The hybridized array was washed, fixed, and scanned using an Axon GenePix 4000B Microarray Scanner (Molecular Devices Corporation, Sunnyvale, CA, USA) [19].

\section{miRNA labeling and array hybridization}

RNA labeling and array hybridization were performed as per Exiqon's manual. After quality control, a miRCURY ${ }^{\top M}$ Hy3/Hy5 Power Labeling Kit (Exiqon, Vedbaek, Denmark) was used according to the manufacturer's guidelines for miRNA labeling by the following steps: first, $1 \mu \mathrm{L}$ RNA in $2.0 \mu \mathrm{L}$ water was combined with $1.0 \mu \mathrm{L}$ CIP buffer and CIP (Exiqon), the mixture was incubated for $30 \mathrm{~min}$ at $37^{\circ} \mathrm{C}$, and the reaction was terminated by incubation for $5 \mathrm{~min}$ at $95^{\circ} \mathrm{C}$. Then, $3.0 \mu \mathrm{L}$ labeling buffer, $1.5 \mu \mathrm{L}$ fluorescent label ( $\mathrm{Hy}^{\mathrm{TM}}{ }^{\mathrm{TM}}$ ), $2.0 \mu \mathrm{L}$ DMSO, and $2.0 \mu \mathrm{L}$ labeling enzyme were added to the mixture. The labeling reaction was incubated for $1 \mathrm{~h}$ at $16^{\circ} \mathrm{C}$ and terminated by incubation for $15 \mathrm{~min}$ at $65^{\circ} \mathrm{C}$. After stopping the labeling

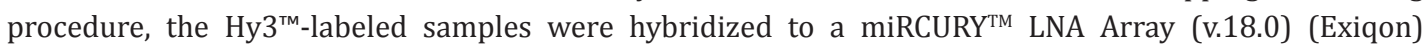
according to the manufacturer's instructions. A total of $25 \mu \mathrm{L} \mathrm{Hy} 3^{{ }^{\mathrm{TM}}}$-labeled sample was mixed with 25 $\mu \mathrm{L}$ hybridization buffer and denatured for $2 \mathrm{~min}$ at $95^{\circ} \mathrm{C}$, incubated on ice for $2 \mathrm{~min}$, and hybridized to the microarray for $16-20 \mathrm{~h}$ at $56^{\circ} \mathrm{C}$ in a 12-Bay Hybridization System. Following hybridization, the slides were washed several times using a Wash Buffer Kit (Exiqon). The slides were scanned using an Axon GenePix 4000B Microarray Scanner (Axon Instruments, Foster City, CA, USA) [20].

\section{Microarray data analysis}

The circRNA array scanned images were imported into GenePix Pro 6.0 software (Axon) for grid alignment and data extraction. Quantile normalization of raw data and subsequent data processing were performed using the R software package. The circRNAs that were flagged as "expressed" ( $>2$-fold background standard deviation) in at least 2 of 6 samples were retained for further differential analyses. Differentially expressed circRNAs were identified through absolute fold change, and $P$-values were calculated using Student's t-test (independent samples). An absolute fold change $\geq 2.0$ and $P<0.05$ were selected as thresholds for significant differential expression of circRNAs.

The miRNA array scanned images were also imported into GenePix Pro 6.0 software (Axon) for grid alignment and data extraction. Replicated miRNAs were averaged, and miRNAs with an intensity $\geq 30$ in all samples were chosen to calculate the normalization factor. Expressed data were normalized using median normalization. After normalization, miRNAs that were significantly differentially expressed between both groups were identified using fold change and $P$-value. An absolute fold change $\geq 1.5$ and $P<0.05$ were selected as thresholds for significant differential expression of miRNAs. Finally, hierarchical clustering was performed to show distinguishable miRNA expression profiling among the samples.

\section{Bioinformatics analysis}

Recent evidence has demonstrated that circRNAs play a crucial role in fine tuning the level of miRNAmediated regulation of gene expression by sequestering miRNAs [21]. To explore the relationship between circRNAs and miRNAs, we predicted their interactions using Arraystar's home-made miRNA target prediction software, which is based on TargetScan and miRanda, and annotated the details with a twodimensional (2D) structural diagram from which the strength of binding can be estimated [21].

\section{Quantitative real-time PCR}

Four differentially expressed miRNAs (miR-467d-3p, miR-491-5p, miR-3098-3p, and miR-3103-5p), which were predicted to bind to differentially expressed circRNAs, were validated by quantitative real-time PCR (qRT-PCR) using a ViiA ${ }^{\text {TM }} 7$ Real-Time PCR System (Applied Biosystems) in another 5 pairs of heart samples from the alcohol-treated and control groups. Total RNA was extracted using the TRIzol reagent (Invitrogen, CA, USA), and cDNA was synthesized from $1.0 \mu \mathrm{g}$ total RNA using the Gene Amp PCR System 9700 (Applied Biosystems; primers were designed and synthesized for 4 miRNAs). The expression of each miRNA was represented relative to the expression of U6 small nuclear RNA as an internal control. For 


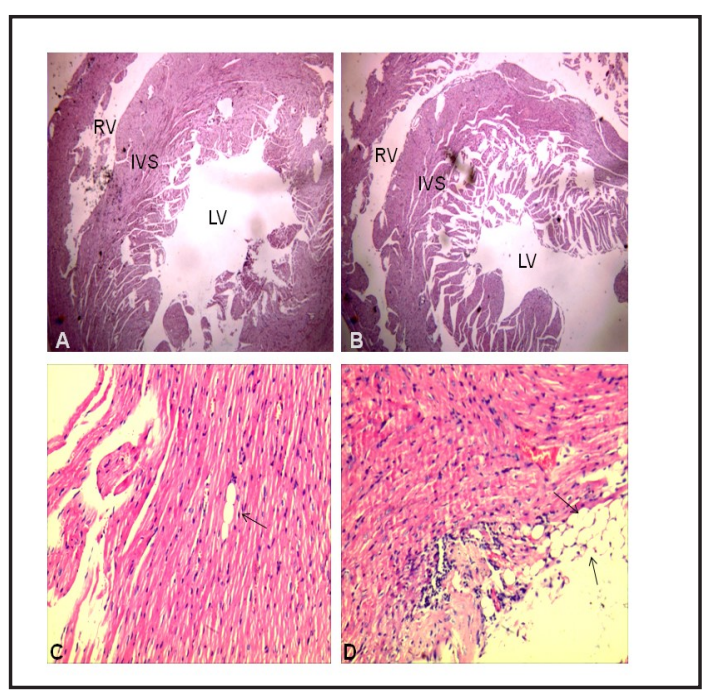

Fig. 1. Cardiac histology. The hearts of control mice (A) and alcohol-treated mice (B) were sectioned at the same level and stained with H\&E (40x magnification). Interventricular septal thickness was increased in alcohol-treated hearts compared with control hearts. A small number of tiny fat vacuoles were observed in control hearts, while both their size and number increased in alcohol-treated hearts (100× magnification). LV, left ventricle; RV, right ventricle; IVS, interventricular septum; arrow, fat vacuoles.

quantitative results, the expression of each miRNA was represented as fold change using the $2^{-\Delta \Delta \mathrm{Ct}}$ method. Differences in miRNA expression between the alcohol-exposed and control hearts were analyzed using Student's t-test with SPSS (Version 16.0; SPSS, Inc.). $P$-values $<0.05$ were considered significant.

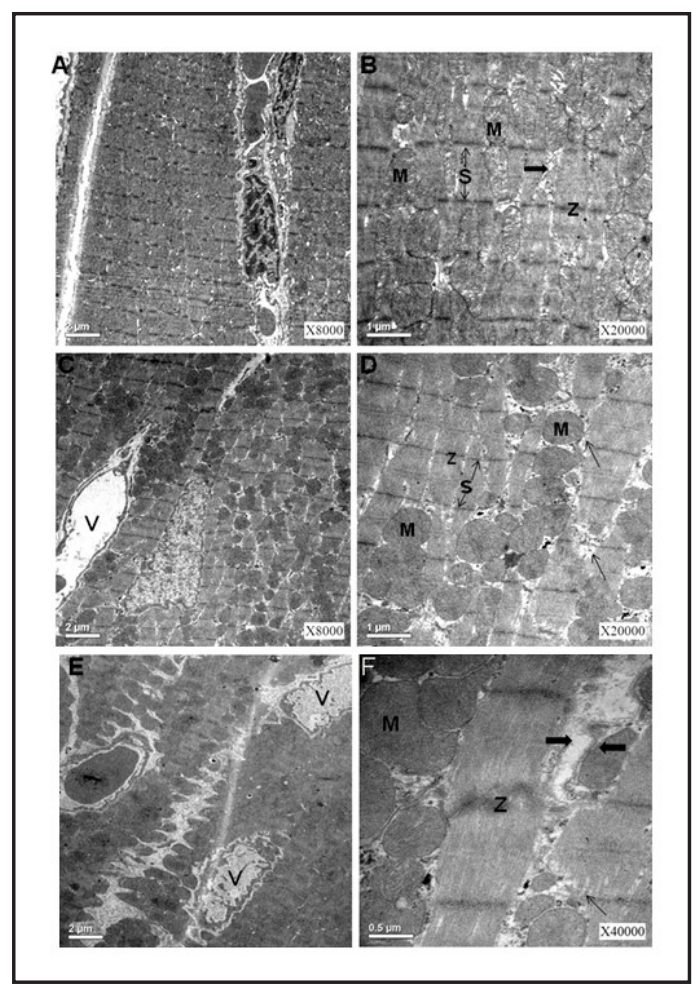

Fig. 2. Transmission electron micrographs of cardiomyocytes. Cardiomyocytes in control group mice (A, B) show normal ultrastructural features. While cardiomyocytes from alcohol-treat mice show an increased number of swollen mitochondria and large vacuoles (C); myofibrillar lysis (arrow) and swollen mitochondria with disorganized and degenerated cristae (D); large vacuoles (V) with a double membrane (E); and dilated sarcoplasmic reticulum (arrowhead; F). M, mitochondrion; S, sarcomere; Z, Z-line; V, vacuole. All magnifications are indicated with reference bars.

\section{Results}

\section{Histological and ultrastructural changes of alcohol-fed mice}

Twenty-four weeks of alcohol consumption had no influence on body weight and heart weight, although it significantly exacerbated alcohol-induced interventricular septum hypertrophy. There was no significant difference in mean body weight or heart weight-tobody weight ratio between the alcohol-fed and control groups.

We identified intra-myocardial changes by H\&E staining and transmission electron microscopy. Evaluations of H\&E-stained paraffin sections using light microscopy demonstrated that the thickness of the interventricular septum was significantly increased in alcohol-exposed hearts compared with control hearts at the same cross-sectional level (Fig. 1A, B). At high power (100× magnification), lipids were found to accumulate in alcoholexposed cardiomyocytes as vacuoles; both their number and size were increased in alcoholtreated hearts compared with control hearts (Fig. 1C, D). Transmission electron microscopy revealed that cardiomyocytes of the control group exhibited fine structural characteristics considered typical for the heart (Fig. 2A, B). In contrast, the alcohol-exposed group showed

\section{KARGER}


considerable myofibrillar alterations, including fragmented and disarrayed myofibrils in cardiomyocytes, vacuolization of cardiomyocytes, dilated sarcoplasmic reticulum, and an increased number of swollen mitochondria with disorganized and degenerated cristae (Fig. $2 \mathrm{C}-\mathrm{F}$ ). The increase in the number of mitochondria and mitochondrial alterations per cell was particularly typical. In some cardiomyocytes, the mitochondria occupied a larger area than the myofibrils (Fig. 2C). The morphological changes and ultrastructural damage seen in the alcohol-treated mice were similar to many of those seen in biopsy specimens from human patients with ACM [22]. These results indicated that we had successfully established a chronic alcohol-induced ACM model.

\section{Differentially expressed circRNAs}

As a preliminarily exploration of the biological significance of circRNAs in ACM, circRNA expression profiles were determined in the myocardia of mice after alcohol consumption for 24 weeks through microarray analysis. The mouse circRNA microarray contains 1797 circRNA probes collected from stringent experimentally validated conditions that are curated carefully from landmark publications, allowing the systematic profiling of the circRNA transcriptome in physiological and pathophysiological conditions. In the circRNA expression profiling data, we found a total of 643 circRNAs expressed in the mouse LV myocardium. Using these data, we compared circRNA expression levels between 3 alcohol-exposed hearts and 3 control hearts and identified an average of 114 up-regulated circRNAs and 151 downregulated circRNAs that were significantly differentially expressed (absolute fold change $\geq$ $2.0, P<0.05$ ). A scatter plot was used to visualize and assess circRNA expression variation between both groups (Fig. 3A). A box plot was used as a convenient and quick method to visualize and compare the distributions of the expression values of the circRNA profiles

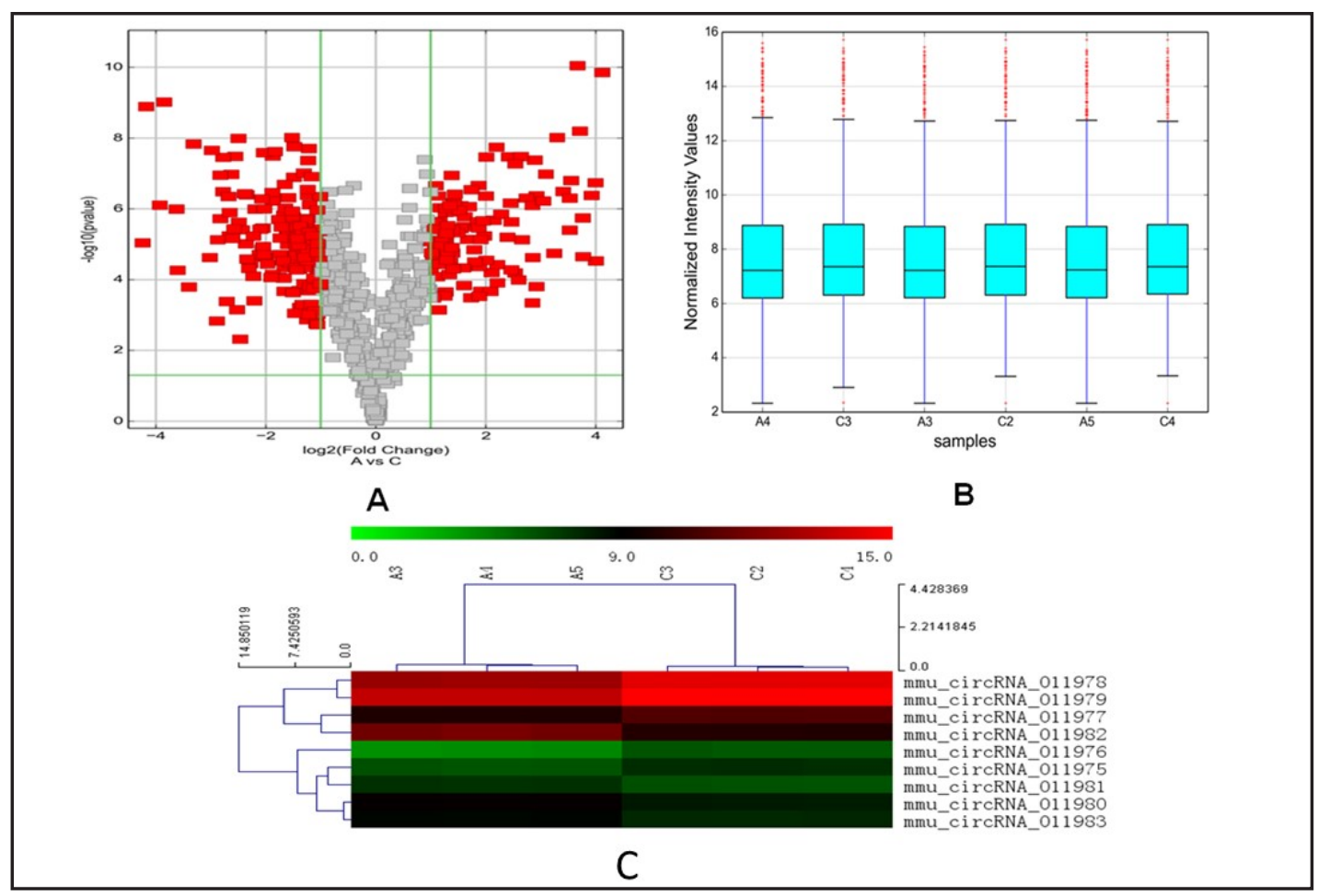

Fig. 3. The expression profiles of circRNAs were compared between alcohol-treated and normal hearts. A: Volcano plots of the circRNA expression profile; red dots represent circRNAs with a 2-fold change in expression $(\mathrm{P}<0.05)$. B: Normalized circRNA profile box plot. C: Hierarchical clustering of distinguishable circRNA expression profiling among samples. Red represents high expression, while green represents low expression.

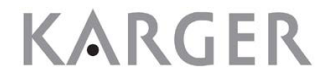




\section{Cellular Physiology Cell Physiol Biochem 2018;46:2284-2296 and Biochemistry Published onIIne: May 07, $2018 \quad \begin{aligned} & \text { DOI: 10.1159/000489596 } 2018 \text { The Author(s). Published by S. Karger AG, Basel } \\ & \text { www.karger.com/cpb }\end{aligned}$ \\ Yang et al.: Circular RNAs and MicroRNAs Profile in ACM Mouse Hearts}

for the samples after normalization (Fig. 3B). Clustering analysis showed the relationships among the circRNA expression patterns that were present in the samples (Fig. 3C).

\section{Differentially expressed miRNAs}

From the miRNA expression profiling data, a total of 1174 miRNAs were identified in heart samples. Among the 3 alcohol-treated myocardia and 3 control myocardia, an average of 70 miRNAs were expressed at a level above background on the microarray and 28 miRNAs were expressed under background level (absolute fold change $\geq 1.5$ ). Of these data, an average of 11 miRNAs was significantly differentially up-regulated and 8 miRNAs were significantly differentially down-regulated (absolute fold change $\geq 1.5, P<0.05$ ). A scatter plot was used to assess miRNA expression variation between both groups (Fig. 4A). A box plot was used to visualize the distribution of the miRNA profiles. Clustering analysis also showed the presence of relationships among the miRNA expression patterns in the samples (Fig. 4B).

\section{miRNA target sites in differentially expressed circRNAs}

Through bioinformatics analysis, more than 5 different MREs were predicted in most of the differentially expressed circRNAs (a total of 249 out of 265 differentially expressed circRNAs), which means that each circRNA could bind to more than 5 different miRNAs. Among the predicted miRNA dataset, there were 4 miRNAs that were from the differentially expressed miRNA profile: miR-467d-3p, miR-491-5p, miR-3098-3p, and miR-3103-5p. More specifically, that means circRNA_012141 contains an MRE that could interact with miR-467d-3p (Fig. 5A); circRNA_014561 contains 4 different MRE sites that could interact with miR-491-5p (Fig. 5B); there are 3 different circRNAs each containing an MRE that could theoretically interact with miR-3098-3p, namely, circRNA_000043, circRNA_011514, and circRNA_011975 (Fig. 5C). There are 4 different circRNAs that could interact with

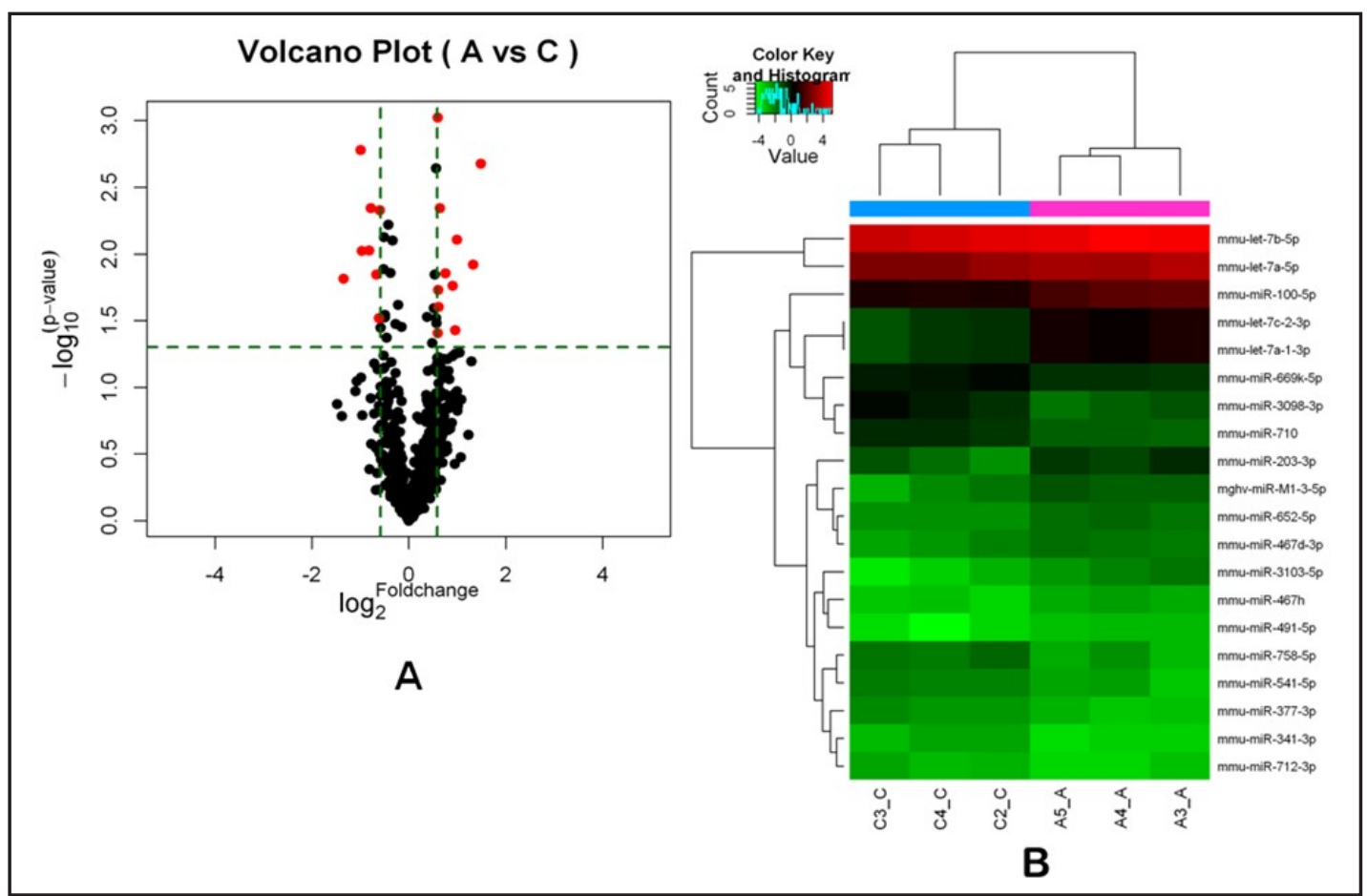

Fig. 4. The miRNA expression profiles were compared between alcohol-treated and normal hearts. A: Volcano plot of the miRNA expression profile; red dots represent miRNAs with a 1.5 -fold change in expression $(\mathrm{P}<0.05)$; B: Hierarchical clustering of distinguishable miRNA expression profiling among samples. Red represents high expression, while green represents low expression. 
miR-3103-5p: circRNA_000224, circRNA_001397, circRNA_012342, and circRNA_017811. circRNA_000224 contains 3 MREs that could interact with miR-3103-5p. circRNA_001397 and circRNA_012342 both contain 2 MREs and circRNA_017811 contains an MRE that could interact with miR-3103-5p (Fig. 5D).

There were 5 types of seed-matched sites between these 4 miRNAs and 9 circRNAs. They are the 7mer-m8 site, which perfectly matches positions 2-8 of the mature miRNA (the seed + position 8); the offset 6mer site, which perfectly matches positions 3-8 of the miRNA; the imperfect site, where positions 2-7 of the miRNA have a G:U non-standard pairing or mismatch or are missing; the 8mer site, which perfectly matches positions 2-8 of the mature miRNA (the seed + position 8) followed by an "A"; and the 7mer-A1 site, which perfectly matches positions 2-7 of the mature miRNA (the seed) followed by an " $A$ ". The 2D structures of those 4 miRNA-binding sites on the 9 differentially expressed circRNAs are listed in Fig. 5.

\section{qRT-PCR validation}

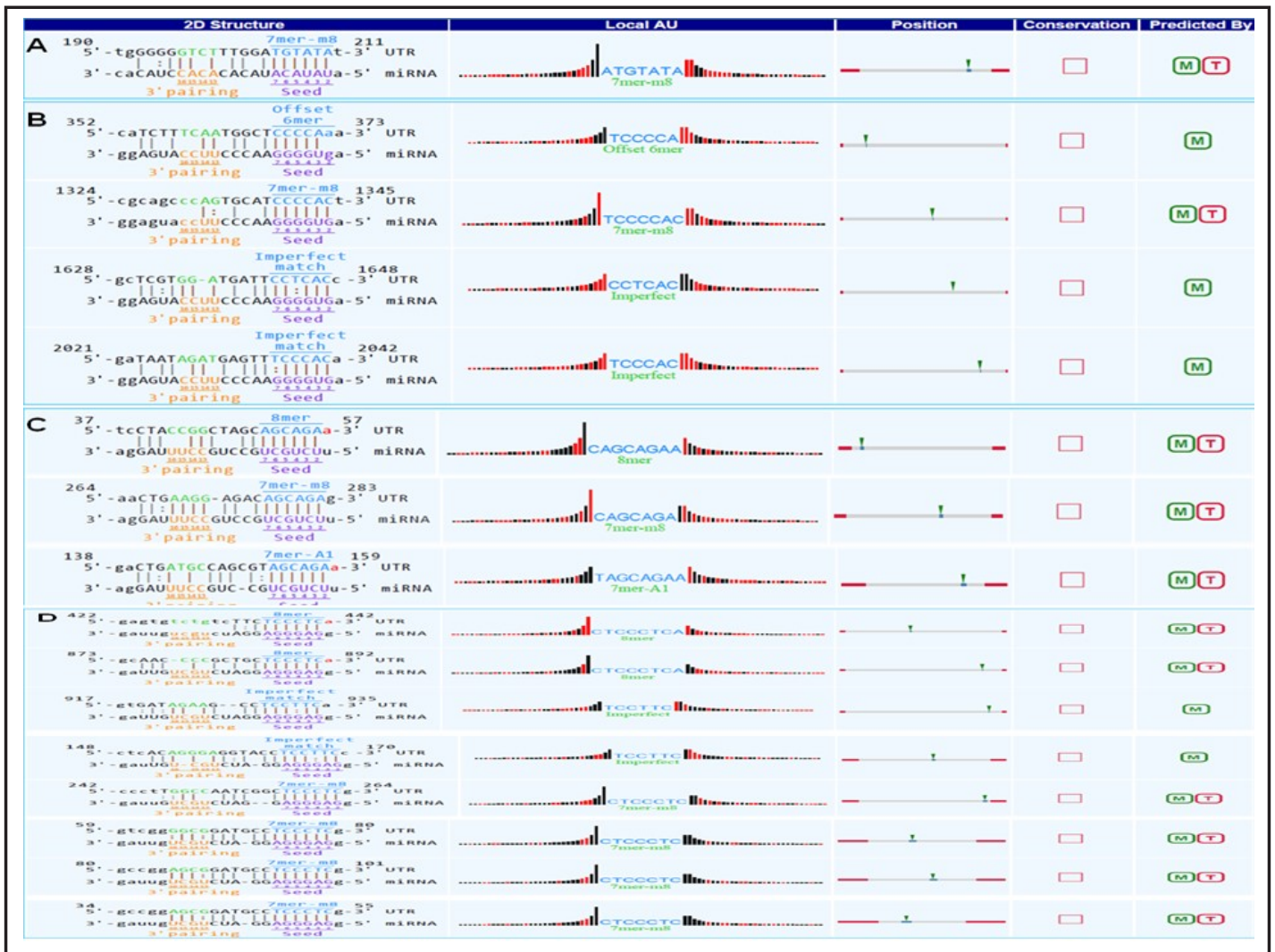

Fig. 5. The 2D structure of the miRNA-binding sites on circRNAs. 2D Structure: the specific location of the binding sites on the full-length sequence of circRNA and the types of binding site. "I" indicates an exact match; ":" indicates G:U and T:G pairing. The base pairing at 2-7 and 13-16 are particularly important for site recognition, highlighted in brown. Local AU: weighting of the AU. This feature affects the accessibility of the binding sites. The red bar indicates that the location is A: $U$. The redder the bar is, the higher the weighting of the AU. Position: relative position of the binding sites on circRNA. Conservation: relatively conservative estimates of the seed complementary region and untranslated region (UTR) between species. Currently, data for constructing a phylogenetic tree of different species of circRNAs are not sufficient. The "conservation" section is unknown for circRNAs. Predicted By: whether this locus is in accordance with the threshold criteria of the prediction algorithms (miRanda and TargetScan). A: The 2D structure of miR-467d3p on circRNA_012141. B: The 2D structure of miR-491-5p on circRNA 014561. C: The 2D structure of miR3098-3p on circRNA_000043, circRNA_011514, and circRNA_011975. D: The 2D structure of miR-3013-5p on circRNA_000224, circRNA_001397, circRNA_012342, and circRNA_017811. 


\section{Cellular Physiology Cell Physiol Biochem 2018;46:2284-2296 \\ \begin{tabular}{l|l} 
DOI: $10.1159 / 000489596$ \\
and Biochemistry
\end{tabular} $\begin{aligned} & \text { Published onnine:May 01, } 2018 \\
& \text { www.karger.com/cpb }\end{aligned}$ \\ Yang et al.: Circular RNAs and MicroRNAs Profile in ACM Mouse Hearts}

Fig. 6. Comparison between the microarray data and qRTPCR results for miRNAs. miR-467d-3p, miR-491-5p, and miR3098-3p that were determined to be differentially expressed in alcohol-treated myocardium compared with control myocardium in 5 samples by microarray analysis were validated by qRT-PCR. Whereas, the validation results for miR-3013-5p indicated no significant difference in expression fold-change between the alcohol-treated and control groups. The heights of the columns in the chart represent fold change (alcohol/control) in expression across the 5 samples for each of the 4 validated miRNAs; the bars represent standard errors.

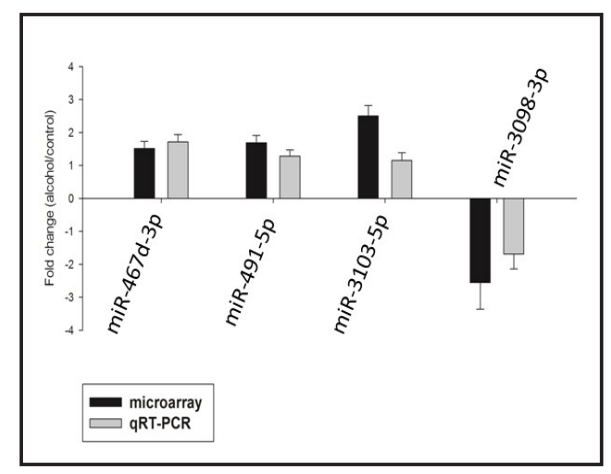

We performed qRT-PCR assays to confirm the expression pattern of the 4 differentially expressed miRNAs. The results demonstrated that miR-467d-3p and miR-491-5p were upregulated and miR-3098-3p was down-regulated in the alcohol-exposed myocardial samples compared with the control samples $(P<0.05)$. The qRT-PCR data demonstrated strong consistency with the microarray data, whereas there was no significant difference in the expression level of miR-3103-5p between the alcohol-treated and control groups (Fig. 6).

qRT-PCR analysis of the circRNAs revealed that a 1.96-fold down-regulation (2.56fold in the microarray analysis) was observed for circRNA_011975, which was the only one consistent with the microarray analysis. The qRT-PCR expression pattern for circRNA_014561 and circRNA_011514 was completely reversed compared with that of the microarray analysis; a 2.63-fold down-regulation (2.15-fold up-regulation in the microarray analysis) was observed for circRNA_014561, and a 1.11-fold down-regulation (2.22-fold upregulation in the microarray analysis) was observed for circRNA_011514. We were unable to amplify circRNA_000043 by PCR.

\section{Discussion}

Chronic alcohol consumption is a significant risk factor that promotes cardiac pathology and dysfunction. Chronic alcohol consumption leads to a number of alterations in the metabolic function of the heart $[2,23]$ and results in a deterioration of heart function [6, 24$26]$, which is a leading cause of cardiomyopathy. The exact amount and duration of alcohol consumption associated with the development of ACM is humans are variable. ACM is highly associated with race, gender, genetic predisposition, and lifestyle factors [27]. Excessive and prolonged consumption of $\geq 100 \mathrm{~g}$ alcohol a day in men and $80 \mathrm{~g}$ alcohol a day in women over a period of 10 years leads to the development of ACM [24, 28, 29]. In the present study, we used an alcohol ad libitum consumption scenario for 24 weeks in order to eliminate the presence of stress caused by intravenous administration or aerosolized inhalation forcedfeeding regimens [30]. The results from our study demonstrated that murine myocardia underwent a considerable number of adverse ultrastructural, structural, and histological changes following chronic alcohol consumption. In the present study, myocardial changes were found in the chronic alcohol-fed mice, such as fatty acid accumulation, cardiomyocyte vacuolization, myocardial myofibril loss and disarray, sarcoplasmic reticulum edema, and swollen disrupted mitochondria, which are consistent with those of previous reports [3133]. The mechanisms underlying ACM include oxidative stress [4, 5], impaired mitochondrial bioenergetics [2], and derangements in fatty acid metabolism and transport [6]. Our observations in the alcohol-treated mice using light and electron microscopy are in line with those seen in postmortem myocardial biopsies from human subjects with ACM [22, 34], even though we have no evidence that our mice developed dilated cardiomyopathy (DCM). On the contrary, we observed significant interventricular septum hypertrophy in the alcoholexposed hearts, which is consistent with previous findings that chronic alcohol consumption may lead to cardiac remodeling and cardiac hypertrophy [35]. Thus, this murine model 
recapitulates important features of human ACM.

The metabolic basis of ACM is probably multi-factorial, but alterations in the expression of myocardial genes are also main contributing factors for the precipitation and progression of this disease. ACM is a specific DCM secondary to a history of long-term heavy alcohol consumption; in other words, ACM is a common cause of DCM. Long-term alcohol abuse in patients with DCM accelerates their condition and leads to death, whereas abstinent patients with DCM have beneficial changes in LV function [36]. The pathophysiologic hallmarks of both ACM and DCM are similar and include enlarged ventricular dimensions, normal or reduced $L V$ wall thickness, increased LV mass, and finally death from congestive heart failure.

Genetic mutations account for a significant percentage of cardiomyopathies. miRNAs have emerged as powerful post-transcriptional regulators of gene expression, especially in the regulation of several aspects of cardiomyopathy. Different miRNA expression patterns have been described for DCM in humans; for example, let-7a, let-7b, let-7c, and miR-100 are up-regulated in LV samples from patients with DCM compared with healthy controls [37]. The miRNA profile of DCM is largely concordant with the differentially expressed miRNA profile of the present study, in which let-7a-5p, let-7b-5p, let-7c-2-3p, and miR-100-5p were up-regulated in the alcohol-treated group compared to the control group. Let- 7 and its family members are highly conserved across species in terms of sequence and function. The up-regulation of the let-7 family may help to increase myocardial fibrosis and apoptosis, as cardiomyocytes in which let-7 is inhibited have a decreased number of apoptotic cells and reduced myocardial fibrosis [38]. Sucharov et al. [39] also reported that miR-100 was up-regulated in failing DCM hearts and demonstrated that miR-100 has a specific role in the regulation of the adult isoforms of cardiac genes .

The observed changes in the expression levels of individual miRNAs are functionally relevant, albeit the magnitude of the change in their expression was generally small. However, miRNA activity has also been shown to be affected by the presence of miRNA sponge transcripts $[40,41]$. In recent years, a growing number of studies have found that circRNAs are an important class of miRNA-dependent post-transcriptional regulators [11, 12] that play a crucial role in fine tuning the level of miRNA-mediated regulation of gene expression by sequestering miRNAs [17]. Almost all of these previous studies identified circRNAs and quantified their relative abundance by high-throughput RNA sequencing from non-polyadenylated RNA transcripts and revealed their potential biological function as miRNA sponges.

In this study, we utilized a different screening method to identify the circRNA profile, namely, a circRNA microarray. This circRNA microarray, which was developed by Arraystar, is represented by a splice junction probe that can identify individual circRNAs reliably and accurately, even in the presence of their linear counterparts. From the microarray expression profiles, we identified a total of 643 circRNAs that were expressed in the mouse LV myocardium; 114 up-regulated circRNAs and 151 down-regulated were significantly differentially expressed $(\geq 2.0$-fold, $P<0.05$ ) in all alcohol-treated hearts. This tremendous change of circRNA expression in the heart was the result of heavy alcohol consumption, further indicating that circRNAs might play significant biological roles in the development of ACM.

Additionally, all of the differentially expressed circRNAs were annotated with potential target sites for miRNAs, which are referred to as MREs. An MRE is a kind of critical sequence that is helpful for unraveling the function of circRNAs as natural miRNA sponges. We predicted the interaction between differentially expressed miRNAs and differentially expressed circRNAs using Arraystar's home-made miRNA target prediction software, which is based on TargetScan and miRanda. More than 5 MREs were predicted in most of the differentially expressed circRNAs. An increasing number of studies has revealed that circRNAs may be new protagonists of the ceRNA network that function as a natural sponge to titrate miRNAs and prevent them from binding to mRNAs, according to the ceRNA hypothesis $[11,12]$, in which MREs regulate miRNA expression levels [10]. Although more than 5 MREs were predicted to be contained in the same circRNA or more than 3 MREs were predicted to bind 
to the same miRNA, not all MREs on circRNA can bind to the corresponding miRNA; their relative concentration and specific nucleotide composition may be partially different and the effectiveness of each MRE in binding to an miRNA is critical for overall ceRNA function [10].

Although an increasing number of studies have found that circRNAs are implicated in the development of several different diseases, such as atherosclerosis, nervous system disorders, and cancers $[11,18,42,43]$, their potential biological functions are significantly underestimated. CircRNAs carrying several different MREs make miRNA regulation complicated. A circRNA can be used to block an entire miRNA seed family based on its natural miRNA sponge potential. The stability and conserved properties of circRNAs are providing inspiration for the engineering of gene vector-encoded sponges as potent inhibitors of miRNA activity [44]. In contrast, Hansen et al. [45] found that circRNAs could also be cleaved by miRNAs in an Ago2-slicer-dependent manner, which further adds to the complexity of the interaction between circRNAs and miRNAs. In recent years, methods for the identification of circRNAs have been developed, including molecular methods and genome-wide approaches, such as RNA-seq and microarray analysis. Data from recent genome-scale studies of circRNAs have provided strong evidence for the presence of thousands of circRNAs in diverse human cell types $[11,14,15]$, even in human plasma and cell-free saliva from healthy individuals [46], and their transcription levels vary according to cell type [42, 47]. These properties provide circRNAs with the potential to become ideal biomarkers for the diagnosis of a wide range of diseases.

As a recent addition to the growing list of non-coding RNAs [48], no doubt more examples and even additional roles will be found for circRNAs. Although we have predicted that the binding of miRNAs to circRNAs may help to infer the function of circRNAs, the findings described in this article are merely a starting point for the study of circRNAs. Our conclusions were obtained only through the changes in their levels and bioinformatics analysis, which need experimental identification and validation. Thus, functional experiments are warranted to investigate thoroughly the mechanism of the interaction between circRNAs and miRNAs in ACM.

\section{Acknowledgements}

This work was supported by the National Natural Science Foundation of China (81160035 and 81460044), Yunnan Provincial Science and Technology Department (2014HB031 and 2017FE468 (-039)) and Yunnan Health Training Project of High Level Talents.

\section{Disclosure Statement}

The authors declare that no conflict of interests exists.

\section{References}

1 Hookana E, Junttila MJ, Kaikkonen KS, Ukkola O, Kesaniemi YA, Kortelainen ML, Huikuri HV: Comparison of family history of sudden cardiac death in nonischemic and ischemic heart disease. Circ Arrhythm Electrophysiol 2012;5:757-761.

2 Guo R, Ren J: Alcohol dehydrogenase accentuates ethanol-induced myocardial dysfunction and mitochondrial damage in mice: role of mitochondrial death pathway. PLoS One 2010;5:e8757.

3 Preedy VR, Patel VB, Reilly ME, Richardson PJ, Falkous G, Mantle D: Oxidants, antioxidants and alcohol: implications for skeletal and cardiac muscle. Front Biosci 1999;4:e58-66.

4 Jing L, Jin CM, Li SS, Zhang FM, Yuan L, Li WM, Sang Y, Li S, Zhou LJ: Chronic alcohol intake-induced 


\section{Cellular Physiology Cell Physiol Biochem 2018;46:2284-2296 \begin{tabular}{l|l} 
DOI: 10.1159/000489596 & O 2018 The Author(s). Published by S. Karger AG, Basel \\
www.karger.com/cpb
\end{tabular}}

Yang et al.: Circular RNAs and MicroRNAs Profile in ACM Mouse Hearts

oxidative stress and apoptosis: role of CYP2E1 and calpain-1 in alcoholic cardiomyopathy. Mol Cell Biochem 2012;359:283-292.

5 Otis JS, Brown LA, Guidot DM: Oxidant-induced atrogin-1 and transforming growth factor-beta1 precede alcohol-related myopathy in rats. Muscle Nerve 2007;36:842-848.

6 6 Hu C, Ge F, Hyodo E, Arai K, Iwata S, Lobdell Ht, Walewski JL, Zhou S, Clugston RD, Jiang H, Zizola CP, Bharadwaj KG, Blaner WS, Homma S, Schulze PC, Goldberg IJ, Berk PD: Chronic ethanol consumption increases cardiomyocyte fatty acid uptake and decreases ventricular contractile function in C57BL/6J mice. J Mol Cell Cardiol 2013;59:30-40.

-7 De Rosa S, Curcio A, Indolfi C: Emerging role of microRNAs in cardiovascular diseases. Circ J 2014;78:567575.

8 Shukla GC, Singh J, Barik S: MicroRNAs: Processing, Maturation, Target Recognition and Regulatory Functions. Mol Cell Pharmacol 2011;3:83-92.

-9 Dai Q Li J, Zhou K, Liang T: Competing endogenous RNA: A novel posttranscriptional regulatory dimension associated with the progression of cancer. Oncol Lett 2015;10:2683-2690.

10 Salmena L, Poliseno L, Tay Y, Kats L, Pandolfi PP: A ceRNA hypothesis: the Rosetta Stone of a hidden RNA language? Cell 2011;146:353-358.

11 Memczak S, Jens M, Elefsinioti A, Torti F, Krueger J, Rybak A, Maier L, Mackowiak SD, Gregersen LH, Munschauer M, Loewer A, Ziebold U, Landthaler M, Kocks C, le Noble F, Rajewsky N: Circular RNAs are a large class of animal RNAs with regulatory potency. Nature 2013;495:333-338.

12 Hansen TB, Jensen TI, Clausen BH, Bramsen JB, Finsen B, Damgaard CK, Kjems J: Natural RNA circles function as efficient microRNA sponges. Nature 2013;495:384-388.

13 Jeck WR, Sorrentino JA, Wang K, Slevin MK, Burd CE, Liu J, Marzluff WF, Sharpless NE: Circular RNAs are abundant, conserved, and associated with ALU repeats. RNA 2013;19:141-157.

14 Wang PL, Bao Y, Yee MC, Barrett SP, Hogan GJ, Olsen MN, Dinneny JR, Brown PO, Salzman J: Circular RNA is expressed across the eukaryotic tree of life. PLoS One 2014;9:e90859.

15 Salzman J, Gawad C, Wang PL, Lacayo N, Brown PO: Circular RNAs are the predominant transcript isoform from hundreds of human genes in diverse cell types. PLoS One 2012;7:e30733.

16 Capel B, Swain A, Nicolis S, Hacker A, Walter M, Koopman P, Goodfellow P, Lovell-Badge R: Circular transcripts of the testis-determining gene Sry in adult mouse testis. Cell 1993;73:1019-1030.

17 Ghosal S, Das S, Sen R, Basak P, Chakrabarti J: Circ2Traits: a comprehensive database for circular RNA potentially associated with disease and traits. Front Genet 2013;4:283.

18 Burd CE, Jeck WR, Liu Y, Sanoff HK, Wang Z, Sharpless NE: Expression of linear and novel circular forms of an INK4/ARF-associated non-coding RNA correlates with atherosclerosis risk. PLoS Genet 2010;6:e1001233.

19 Zhang YG, Yang HL, Long Y, Li WL: Circular RNA in blood corpuscles combined with plasma protein factor for early prediction of pre-eclampsia. Bjog 2016;123:2113-2118.

20 Cai H, Zhou H, Miao Y, Li N, Zhao L, Jia L: MiRNA expression profiles reveal the involvement of miR-26a, miR-548l and miR-34a in hepatocellular carcinoma progression through regulation of ST3GAL5 Lab Invest 2017;97:530-542.

21 Wu HJ, Zhang CY, Zhang S, Chang M, Wang HY: Microarray Expression Profile of Circular RNAs in Heart Tissue of Mice with Myocardial Infarction-Induced Heart Failure. Cell Physiol Biochem 2016;39:205-216.

-22 Tsiplenkova VG, Vikhert AM, Cherpachenko NM: Ultrastructural and histochemical observations in human and experimental alcoholic cardiomyopathy. J Am Coll Cardiol 1986;8:22A-32A.

-23 Hajnoczky G, Buzas CJ, Pacher P, Hoek JB, Rubin E: Alcohol and mitochondria in cardiac apoptosis: mechanisms and visualization. Alcohol Clin Exp Res 2005;29:693-701.

24 Urbano-Marquez A, Fernandez-Sola J: Effects of alcohol on skeletal and cardiac muscle. Muscle Nerve 2004;30:689-707.

25 Kim SD, Bieniarz T, Esser KA, Piano MR: Cardiac structure and function after short-term ethanol consumption in rats. Alcohol 2003;29:21-29.

26 Kim SD, Beck J, Bieniarz T, Schumacher A, Piano MR: A rodent model of alcoholic heart muscle disease and its evaluation by echocardiography. Alcohol Clin Exp Res 2001;25:457-463.

27 Piano MR: Alcoholic cardiomyopathy: incidence, clinical characteristics, and pathophysiology. Chest 2002;121:1638-1650.

28 Lang CH, Frost RA, Summer AD, Vary TC: Molecular mechanisms responsible for alcohol-induced myopathy 


\section{Cellular Physiology Cell Physiol Biochem 2018;46:2284-2296 \begin{tabular}{l|l} 
DOI: 10.1159/000489596 & O 2018 The Author(s). Published by S. Karger AG, Basel \\
www.karger.com/cpb
\end{tabular}}

Yang et al.: Circular RNAs and MicroRNAs Profile in ACM Mouse Hearts

in skeletal muscle and heart. Int J Biochem Cell Biol 2005;37:2180-2195.

29 Player MS, Mainous AG, 3rd, King DE, Diaz VA, Everett CJ: Moderate alcohol intake is associated with decreased risk of insulin resistance among individuals with vitamin D insufficiency. Nutrition 2010;26:100105.

-30 Keane B, Leonard BE: Rodent models of alcoholism: a review. Alcohol Alcohol 1989;24:299-309.

-31 Rubin E: Alcoholic myopathy in heart and skeletal muscle. N Engl J Med 1979;301:28-33.

-32 Teragaki M, Takeuchi K, Takeda T: Clinical and histologic features of alcohol drinkers with congestive heart failure. Am Heart J 1993;125:808-817.

33 Guzzo-Merello G, Cobo-Marcos M, Gallego-Delgado M, Garcia-Pavia P: Alcoholic cardiomyopathy. World J Cardiol 2014;6:771-781.

34 Vikhert AM, Tsiplenkova VG, Cherpachenko NM: Alcoholic cardiomyopathy and sudden cardiac death. J Am Coll Cardiol 1986;8:3A-11A.

-35 Tan Y, Li X, Prabhu SD, Brittian KR, Chen Q, Yin X, McClain CJ, Zhou Z, Cai L: Angiotensin II plays a critical role in alcohol-induced cardiac nitrative damage, cell death, remodeling, and cardiomyopathy in a protein kinase C/nicotinamide adenine dinucleotide phosphate oxidase-dependent manner. J Am Coll Cardiol 2012;59:1477-1486.

36 Gavazzi A, De Maria R, Parolini M, Porcu M: Alcohol abuse and dilated cardiomyopathy in men. Am J Cardiol 2000;85:1114-1118.

-37 Ikeda S, Kong SW, Lu J, Bisping E, Zhang H, Allen PD, Golub TR, Pieske B, Pu WT: Altered microRNA expression in human heart disease. Physiol Genomics 2007;31:367-373.

-38 Tolonen AM, Magga J, Szabo Z, Viitala P, Gao E, Moilanen AM, Ohukainen P, Vainio L, Koch WJ, Kerkela R, Ruskoaho H, Serpi R: Inhibition of Let-7 microRNA attenuates myocardial remodeling and improves cardiac function postinfarction in mice. Pharmacol Res Perspect 2014;2:e00056.

-39 Sucharov C, Bristow MR, Port JD: miRNA expression in the failing human heart: functional correlates. J Mol Cell Cardiol 2008;45:185-192.

40 Moshiri F, Callegari E, D’Abundo L, Corra F, Lupini L, Sabbioni S, Negrini M: Inhibiting the oncogenic mir-221 by microRNA sponge: toward microRNA-based therapeutics for hepatocellular carcinoma. Gastroenterol Hepatol Bed Bench 2014;7:43-54.

41 Ma J, Wu Q Zhang Y, Li J, Yu Y, Pan Q, Sun F: MicroRNA sponge blocks the tumor-suppressing functions of microRNA-122 in human hepatoma and osteosarcoma cells. Oncol Rep 2014;32:2744-2752.

42 Li P, Chen S, Chen H, Mo X, Li T, Shao Y, Xiao B, Guo J: Using circular RNA as a novel type of biomarker in the screening of gastric cancer. Clin Chim Acta 2015;444:132-136.

43 Hansen TB, Kjems J, Damgaard CK: Circular RNA and miR-7 in cancer. Cancer Res 2013;73:5609-5612.

44 Bak RO, Mikkelsen JG: miRNA sponges: soaking up miRNAs for regulation of gene expression. Wiley Interdiscip Rev RNA 2014;5:317-333.

45 Hansen TB, Wiklund ED, Bramsen JB, Villadsen SB, Statham AL, Clark SJ, Kjems J: miRNA-dependent gene silencing involving Ago2-mediated cleavage of a circular antisense RNA. EMBO J 2011;30:4414-4422.

46 Bahn JH, Zhang Q Li F, Chan TM, Lin X, Kim Y, Wong DT, Xiao X: The landscape of microRNA, Piwiinteracting RNA, and circular RNA in human saliva. Clin Chem 2015;61:221-230.

-47 Salzman J, Chen RE, Olsen MN, Wang PL, Brown PO: Cell-type specific features of circular RNA expression. PLoS Genet 2013;9:e1003777.

48 Jeck WR, Sharpless NE: Detecting and characterizing circular RNAs. Nat Biotechnol 2014;32:453-461. 